\title{
Witness the Explosive Cu-Si Alloying Process Inside a TEM
}

\author{
Meng Li, Degang Xie and Zhiwei Shan
}

Center for Advancing Materials Performance from the Nanoscale (CAMP-Nano) \& Hysitron Applied Research Center in China (HARCC), State Key Laboratory for Mechanical Behavior of Materials, Xi'an Jiaotong University, Xi'an 710049, PR China

To achieve higher operation speed, the modern semiconductor industry continues to shrink the size of $\mathrm{Cu}$ interconnects and passes increasingly high current density through the interconnects. In order to guarantee the reliability of these interconnects, keeping the $\mathrm{Cu}$ and $\mathrm{Si}$ from mixing is extremely important since the high diffusivity and reaction activity between $\mathrm{Cu}$ and $\mathrm{Si}$ can greatly endanger the device. Although a thin layer of Ta or TaN is applied to stop the diffusion, there is still a possibility that the $\mathrm{Cu}$ can meet the $\mathrm{Si}$ since the processing procedure isn't perfect. Moreover, the elevated temperature at operating condition can exacerbate the situation. Understanding the failure mechanism is really important for finding an effective remedy. Despite the fact that numerous literatures[1]about the $\mathrm{Cu}-\mathrm{Si}$ diffusion and reaction, direct and complete observation of these phenomenon at the micro- and nanoscale is still rare.

The in-situ TEM technique provide an opportunity to investigate the problem directly at unprecedented spatial and temporal resolution. In this study, we utilized a novel sample design as illustrated in Figure 1 to mimic the real integrated circuit interconnects. The alloying reaction was achieved by applying a high-density current until the explosive $\mathrm{Cu}-\mathrm{Si}$ reaction started. The reaction completed in milliseconds and left a semicircular reaction zone as described in Figure 2. Like how the geological faults revealed the geologic history, the reaction zone showed the whole reaction process. A possible reaction process can be reconstructed for the first time to explain how the interconnect came to failure. The process began with heating up the interconnect, then Copper diffused across the barrier layer and the dielectric layer, and met Silicon. The exothermic alloying reaction then provided more heat to melt the $\mathrm{Cu}$ interconnects, which triggered the explosive alloying reaction [2]. 
References:

[1] A. A. Istratov, E. R. Weber, Journal of the Electrochemical Society, 149(2002), G21-G30

[2] This work was supported by the grants from NSFC (50925104) and 973 Program of China (2010CB631003). The authors thank Dr. Longqing Chen and Dr. Xixiang Zhang from Advanced Nanofabrication, Imaging and Characterization Core Lab, King Abdullah University of Science and Technology are thanked for the sample preparation, the useful discussion as well as the support from Hysitron..

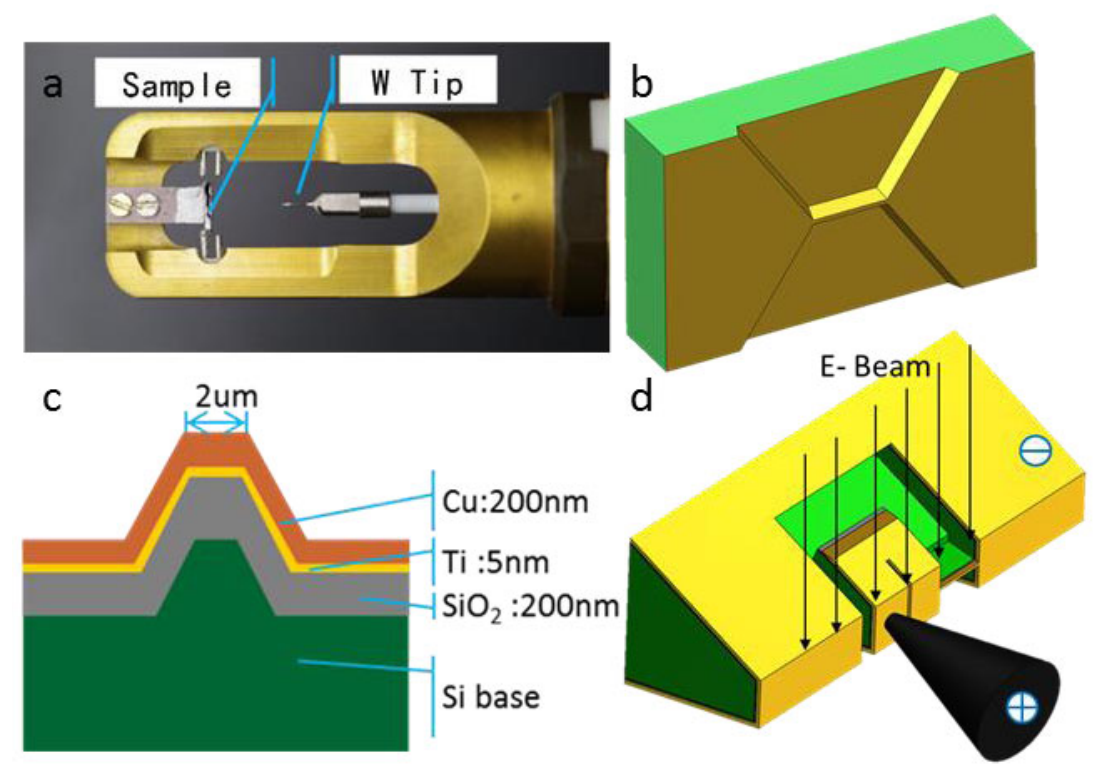

Figure 1. Sample design. (a) Front-end of the Hysitron PI 95 ECR TEM holder; (b) illustration of the silicon wedge sample; (c) illustration of cross-section view of the sample and thickness of each layer; (d) illustration of electrodes and TEM beam direction. The black tip represents the W-tip in (a).
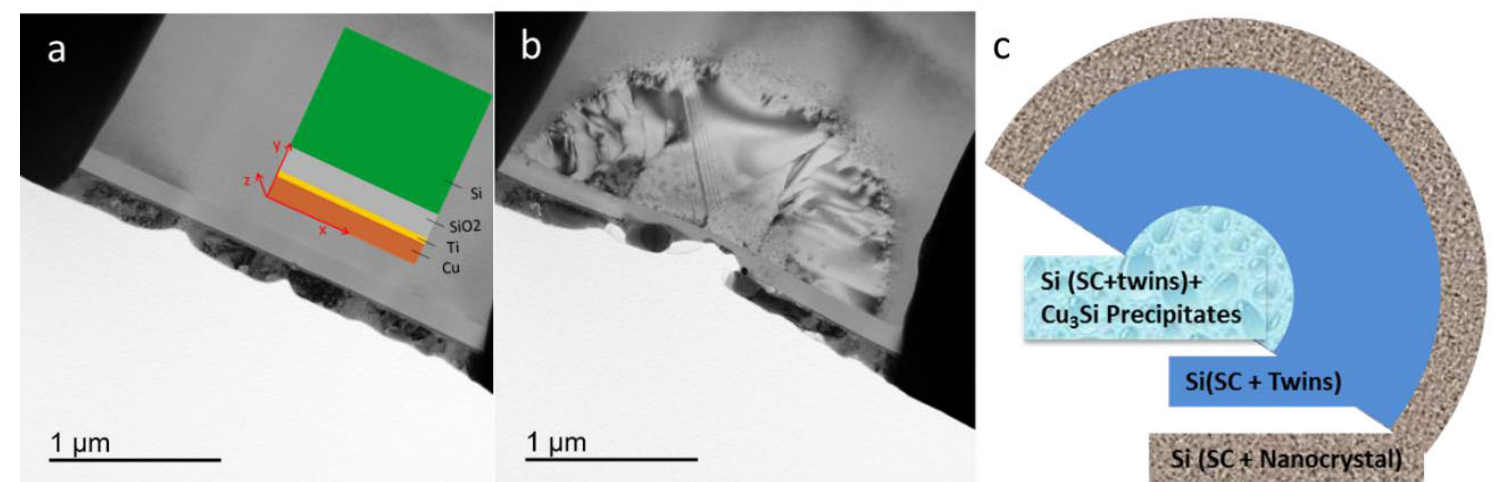

Figure 2. (a) TEM Bright field image of the sample before the experiment, the inset shows component of each layer; (b) TEM Bright field image of the sample after the experiment, the reaction completed in milliseconds. The semicircular pattern with the melting site as its center showed the history of the $\mathrm{Cu}-\mathrm{Si}$ explosive alloying reaction; (c) illustration of component of the 'geological-fault-like' scene. The area closer to the center underwent a longer reaction time, while the brim of the semicircle was still at the initial stage of reaction. This can be explained by the wetting process that started from the melting site, followed by spreading radially like a growing disk. 